\section{How Typhoid Fever is Spread}

ThE case recently reported (NATURE, vol. xiii. p. 33i) by Prof. Frankland from a Swiss village, where the poison of typhoid fever is said "to have filtered through a mile of porous soil, but which had nevertheless lost none of its virulent properties," is certainly so striking that some further reference to authorities seems requisite. If it can be satisfactorily proved that this was the most likely and reasonable origin of the case, it will give additional weight to every endeavour for preserving our water supply from every conceivable impurity; but unless based on the opinion of a competent and skilled investigator of such cases, it will lay us open to the charge of receiving any similar statement that favours our view, however rash. As medical officers of health it is our duty frequently to trace the origin of cases of this disease, and my anxiety to have further information of this case will thus seem reasonable, and I hope will meet with some reply from the distinguished professor.

Rochdale, March

\section{The Ash Seed Screw}

Mr. ALFRED GEORGE ReNSHaw would like to know (NATURE, vol. xiii. p. 367) whether the pitch of the ash seed screw is that which would give most power to the propeller of a steamer.

There is no screw on the samara of the ash (Fraxinus excelsior) while it is green. The pitch of the screw, at the same date, differs on different trees, and also on different seeds of the same tree, and also on different parts of the same seed.

Why the wing generally becomes twisted as it dries is a very interesting question, But what seems to me the most remarkable fact about this phenomenon is, that in every case, and on all trees alike, the thread of the screw is in one direction; that direction being the same as in a cork-screw, or ordinary screwnail.

All varieties of the wild oat (Avena fatua) and the fly oat ( $A v$. sterilis) have long awns, which also in the green stage are straight, but which in ripening become twisted. And in these also, the direction of the screw is uniform and the same as in the ash seed; but the pitch of the screw is variable.

Are these facts the same in other parts of the country? Are the screws left-handed on the solith of the equator?

Summerbill, Aberdeen, March 20 A. Stephen Wilson

\section{OUR ASTRONOMICAL COLUMN}

The Companion of Sirius.-Mr. Wentworth Erck, of Sherrington, Co. Wicklow, in a communication dated March 2I, notes the fact of a considerable diminution in the angle of position of the small star accompanying Sirius, which was detected by Alvan Clark in January, 1862-since the earlier measures, and adds that he cannot now estimate it at more than $55^{\circ}$. This retrograde motion is a consequence of the theory of Dr. Auwers, supposing the comes to be the cause of the anomalous proper motion of Sirius, which has formed the subject of several elaborate memoirs by this eminent astronomer. His last elements of the presumed disturbing body, adapted to the form of double star orbits, are as follow :-

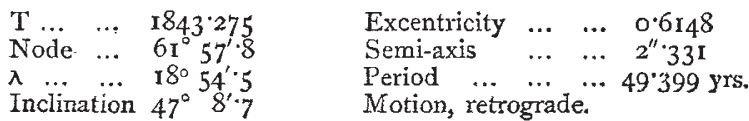

The following angles of position and distances are given by these elements :-

I862.0 Pos. $85^{\circ} \cdot 4$ Dist. I0'10

186.

I868.0 ", 75.0 ", 11 15

$1871^{\circ} 0,70^{\circ} 3, "$ Ir. 20

IS74.0 Pos. $65^{\circ} 5$ Dist. 10"95 $1876.0,62.1,10.59$ $18788^{\circ}, " 5^{\circ} 4$, , 10.05 I $8800^{\circ}, 54.2,9.33$

Dr. Auwers gives a comparison of his calculated angles and distances of the centre of gravity, with those of Clark's companion to 1867 , the agreement being pretty close throughout. But there can be no doubt that the calculation has given the angle too great since that year.
The Washington observations show the following differences:-

$1872.25 \ldots$ Position $(c-0)+5^{\circ} \cdot 6 \ldots$ Distance $(c-0)-0^{\prime \prime} \cdot 40$ $1874.18 \ldots \quad, \quad+6 \cdot 3 \ldots . \quad, \quad-0.46$ $1875^{\circ} 24 \cdots, \quad ", \quad+7 \cdot 2 \ldots . \quad, \quad-0.72$

In the above orbit the limits of distance are $2^{\prime \prime} \cdot 3$ I at I $84 \mathrm{I} \cdot 84$ for a position of $302^{\circ} \cdot 5$, and $\mathrm{II}^{\prime \prime} \cdot 23$ at $1870^{\circ} \mathrm{I} 3$ for position $7 \mathrm{I}^{\circ} .7$, and Auwers remarks that under the former condition the angle changes one degree in ten days, while under the latter condition 233 days are required for the same diminution.

Our correspondent mentions that Lassell's "new star" (Mem. R. Astron. Soc., vol. 36, p. 18), "though exceedingly faint, was distinctly visible. Pos. circa $130^{\circ}$, distance circa $75^{\prime \prime}$," with $7 \frac{1}{2}$ inches aperture power, 200. Lassell, 1865 , January 14 , found the position $127^{\circ} \circ$, distance about one minute.

Dr. Gylden, from the meridian-altitudes of Sirius, observed by Sir Thomas Maclear at the Cape of Good Hope during the years 1836 and 1837 found for the annual parallax of the star o" I93 (Bulletin de I'A cad. de St. Petersbourg, t. vii.). Adopting this value, Dr. Auwers finds for the mean distance between the companion and Sirius, 37 times the earth's mean distance from the sun, and for the masses of Sirius and companion 13.76 and $6 \% 1$ respectively, in units of the sun's mass. The parallax $0^{\prime \prime} \cdot 193$ corresponds to $I, 068,700$ times the sun's distance from the earth.

D'ARrest's COMET.-M. Leverrier's Bulletin International of March 18 , contains the definitive elements obtained by M. Leveau for the next return of this comet to perihelion in 1877 , with an ephemeris for every 20 th day throughout the year, which sufficiently defines the circumstances of the next appearance. The whole of his long-continued investigations relating to the motion of this comet have been conducted by $M$. Leveau with extreme care and minuteness, so that by his investigations, in continuation of those commenced by M. Villarceau, the theory of D'Arrest's comet has been placed upon a similar footing of accuracy to that, upon which the theory of the periodical comet of Faye now stands through the labours of Dr. Axell Möller of Lund.

The next perihelion passage of D'Arrest's comet is found to occur 1877 , May 10.339 M.T. at Paris, and the comet appears to attain its maximum intensity of light about a fortnight subsequently. Other elements of the orbit which apply to 1877 , Jan. I4, are :-

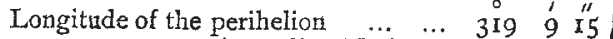

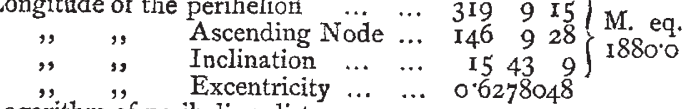

$$
\begin{aligned}
& \text { Logarithm of perihelion distan ... } \\
& \text { Motion direct. }
\end{aligned}
$$

The elements usually found in catalogues of cometary orbits are here substituted for others given by M. Leveau.

The semi-major-axis of the orbit is 3.54139 , to which corresponds a sidereal revolution of $2434^{\circ} 2$ days.

When this comet was last observed by Herr Julius Schmidt at Athens, on December 20, I870, the intensity of light was 0.154 ; the greatest intensity attained in 1877 , about May 26, is only $0^{\circ} 22$, and it is certain that large telescopes will be required for the proper observation of the comet. When theoretically brightest, it rises at Greenwich less than two hours before the sun, and as this difference increases towards the end of the summer, the intensity of light is diminished by one-half. The comet will be nearest to the earth in the middle of October (distance $\approx I^{\circ} 40$ ). M. Leveau promises in a subsequent communication a precise ephemeris for the whole year, and, it may be remarked, it very rarely happens that there is any necessity for predictions extending over so long a period. 